\title{
Prevalência e fatores associados ao uso de psicotrópicos em idosos: uma revisão integrativa
}

\author{
Prevalence and factors associated with the use of psychotropics in the elderly: an integrative review \\ Prevalencia y factores asociados con el uso de psicotrópicos en ancianos: una revisión integrativa
}

Recebido: 09/08/2021 | Revisado: 15/08/2021 | Aceito: 17/08/2021 | Publicado: 21/08/2021

\author{
Jaqueline Maria Silva dos Santos \\ ORCID: https://orcid.org/0000-0003-3690-7811 \\ Centro Universitário Mário Pontes Jucá, Brasil \\ E-mail: jacksil2009@hotmail.com \\ Euda Maria dos Santos Messias \\ ORCID: https://orcid.org/0000-0003-3073-2686 \\ Centro Universitário Mário Pontes Jucá, Brasil \\ E-mail: eudamessias@hotmail.com \\ Raquel Ferreira Lopes \\ ORCID: https://orcid.org/0000-0002-2061-7038 \\ Centro Universitário Mário Pontes Jucá, Brasil \\ E-mail: raquelloppes@gmail.com
}

\begin{abstract}
Resumo
Objetivo: Descrever as dificuldades e as estratégias enfrentadas na utilização de medicamentos controlados entre idosos através de uma investigação na literatura científica disponível. Metodologia: Trata-se de uma revisão integrativa da literatura. Foram utilizadas produções científicas com bases nos periódicos: Scopus, Web of Science e PubMed. Como critérios de inclusão optou-se por artigos originais, publicados entre os anos de 2016 a 2021 , nos idiomas português e inglês, e que abordassem a temática estudada. Utilizando o operador booleano "AND" juntamente com os descritores: Idoso; Psicotrópicos; Prevalência. Resultados: As principais dificuldades e estratégias encontras no presente estudo foi semelhante a vários estudos que relatam a relação entre a utilização dos medicamentos psicotrópicos, onde a grande demanda se encontra associada com a depressão e a baixa qualidade de vida, corrobando com a compreensão sobre os tipos de medicamentos psicofármacos, tempo de duração do tratamento, e a fatores associados que acabam levando a consumação. Conclusão: É necessário que haja uma reestruturação dos serviços de saúde afim de poder oferecer aos pacientes idosos uma maior segurança na conduta terapêutica aplicada, com o intuito de minimizar os efeitos colaterais adversos, orientando sobre o uso correto das medicações de acordo com suas necessidades, através de profissionais especializados e prescritores capacitados.
\end{abstract}

Palavras-chave: Idoso; Psicotrópicos; Prevalência.

\begin{abstract}
Objective: To describe the difficulties and strategies faced in the use of controlled medications among the elderly through an investigation in the available scientific literature. Methodology: This is an integrative literature review. We used scientific productions based on the following journals: Scopus, Web of Science and PubMed. Inclusion criteria included original articles published between 2016 and 2021, in Portuguese and English, and that addressed the theme studied. Using the Boolean operator "AND" together with the descriptors: Elderly; Psychotropic drugs; Prevalence. Results: The main difficulties and strategies found in the present study was similar to several studies that report the relationship between the use of psychotropic medications, where the great demand is associated with depression and low quality of life, corroborating with the understanding about the types of psychotropic medications, duration of treatment, and to associated factors that end up leading to consumption. Conclusion: It is necessary that there is a restructuring of health services in order to offer elderly patients greater safety in the therapeutic conduct applied, in order to minimize adverse side effects, guiding on the correct use of medications according to their needs, through specialized professionals and trained prescribers.
\end{abstract}

Keywords: Elderly; Psychotropics; Prevalence.

\section{Resumen}

Objetivo: Describir las dificultades y las estrategias a las que se enfrenta el uso de medicamentos controlados entre los individuos a través de una investigación en la literatura científica disponible. Metodología: Se trata de una revisión bibliográfica integradora. Se utilizaron productos científicos con base en los periódicos: Scopus, Web of Science y PubMed. Los criterios de inclusión incluyeron artículos originales publicados entre 2016 y 2021, en portugués e inglés, y que abordaran el tema en estudio. Utilizando el operador booleano "AND" junto con los descriptores: Ancianos; Fármacos psicotrópicos; Prevalencia. Resultados: Las principales dificultades y estrategias encontradas en 
el presente estudio fueron similares a varios estudios que reportan la relación entre el uso de psicofármacos, donde la gran demanda está asociada a la depresión y a la baja calidad de vida, corroborando con el conocimiento sobre los tipos de psicofármacos, la duración del tratamiento, y a los factores asociados que terminan llevando al consumo. Conclusión: Es necesario que haya una reestructuración de los servicios de salud para poder ofrecer a los pacientes idosos una mayor seguridad en el tratamiento aplicado, con el objetivo de minimizar los efectos colaterales adversos, orientándose al uso correcto de los medicamentos de acuerdo con sus necesidades, a través de profesionales especializados y prescriptores capacitados.

Palabras clave: Ancianos; Psicotrópicos; Prevalencia.

\section{Introdução}

Globalmente, os países observam uma grande demanda da população idosa com o uso generalizado de drogas, no entanto os efeitos colaterais desses medicamentos acabam acontecendo de forma prejudicial associados a utilização de forma indevida (Secoli, Marquesini, Fabretti, Corona \& Lieber, 2018). Mas, devido à melhoria do diagnóstico das doenças mentais, o surgimento de novos medicamentos no mercado e às novas indicações terapêuticas dos psicotrópicos existentes, o uso de psicofármacos, principalmente antidepressivos, tem aumentado muito (Prado, Francisco \& Barros, 2017).

A sensibilidade do idoso é aumentada, o que significa que sentirá os efeitos do uso de menor quantidade da substância psicoativa consumida, e sua tolerância será reduzida, o que quer dizer que ele não precisará mais usar a substância como antes para obter o mesmo efeito (Sales, Sales \& Casotti, 2017). Ressalta-se que essas substâncias atuam no sistema nervoso central e podem causar mudanças de comportamento, humor e cognição. Eles são produtos químicos que agem e mudam as funções mentais e o estado mental, incluindo medicamentos com efeitos antidepressivos, alucinógenos e/ou sedativos (Prado et al., 2017). Porém, utilizá-lo indiscriminadamente sem o devido acompanhamento acabará por prejudicar a saúde, por serem mais suscetíveis aos agravos (Santos, Viera, Santana, Caldas, Canuto, Araújo \& Lopes, 2020).

No entanto o processo de envelhecimento altera a função e a composição do corpo humano, o que torna necessário ajustar o tipo e a dosagem dos medicamentos (Diniz, Pillon, Monteiro, Pereira, Gonçalves \& Santos, 2017). No Brasil, a taxa de uso de substâncias psicotrópicas encontra-se entre 5,2 e 10,2\%, e a maior parte da população que usa esses medicamentos é idosa (Ackel, Costa L. Costa \& Filho, 2017).

Um estudo realizado em um município da capital de São Paulo, revelou prevalência de 12,2\% de utilização de psicotrópicos entre os idosos residentes no Município de São Paulo: 7,2\% de antidepressivos; 6,1\% de benzodiazepínicos; e $1,8 \%$ de antipsicóticos. Observa-se ainda que $9,1 \%$ dos idosos consumiram um psicotrópico, $2,5 \%$ dois e $0,6 \%$ três agentes. Entre os antidepressivos foram identificados dez representantes (imipramina, clomipramina, amitriptilina, nortriptilina, fluoxetina, citalopram, paroxetina, sertralina, escitalopram e trazodona (Noia, Secoli, Duarte, Lebrão \& Lieber, 2012).

Contudo o uso irracional de substâncias psicotrópicas pode acabar afetando diretamente o tratamento, como por exemplo encobrir sinais e sintomas atribuíveis a estado de ansiedade e/ou depressão, portanto, se faz necessário colaborar com estratégias de intervenção de educação em saúde para os profissionais de saúde (Nascimento \& Salvi, 2018).

Acredita-se que o uso de drogas lícitas por idosos está associado à aceleração da ocorrência de doenças crônicas nesta faixa etária e é considerado um grave problema de saúde pública, causando altos custos sociais sendo um pesado fardo econômico aplicável a contribuintes e agências governamentais (European Monitoring Centre for Drug Addiction, 2010).

Logo, o objetivo geral deste estudo foi descrever as dificuldades e as estratégias enfrentadas na utilização de medicamentos controlados entre idosos através de uma investigação na literatura científica disponível.

\section{Metodologia}

Trata-se de um estudo de forma abrangente de revisão integrativa da literatura conduzido em seis etapas de construção: determinação do tema e seleção de hipóteses, busca na literatura, classificação da pesquisa, avaliação dos estudos 
incluídos, interpretação dos resultados, introdução da revisão. Aponta-se que a contribuição abrangente dos resultados da pesquisa para a inclusão de evidências fornece novos conhecimentos para a prática (Mendes, Silveira \& Galvão, 2019).

Uma revisão integrativa além de identificar as necessidades, fornecem suporte para a tomada de decisões e melhoria da prática clínica como conduzir novas pesquisas para preencher as lacunas no conhecimento científico atual (Crossetti, 2012).

O tema desta pesquisa diz respeito a utilização de medicamentos controlados por pessoas idosas. A partir disto foi elaborada a seguinte questão norteadora: Qual a prevalência e quais fatores estão associados aos idosos que utilizam psicofármacos? Está pergunta se pautou na estratégia PICO (Quadro 1), que diz respeito aos acrônimos das letras, referentes as palavras PICO que representa um acrônimo para Paciente, Intervenção, Comparação e "Outcomes" (desfecho) (Santos, Pimenta \& Nobre, 2019). Salienta-se que não consideramos a vertente "C", visto que este estudo não se propõe ao desenvolvimento de pesquisas clínicas, o que torna dispensável sua utilização (Greenhalgh, 2005).

Quadro 1: Aplicação da estratégia PICO.

\begin{tabular}{|c|c|c|}
\hline Acrônimo & Definição & Aplicação \\
\hline $\mathrm{P}$ & Population & Idoso \\
\hline $\mathrm{I}$ & Intervention & Psicotrópicos \\
\hline $\mathrm{C}$ & Comparation & $\begin{array}{c}\text { Não se aplica por não se tratar de uma pesquisa clínica e sim } \\
\text { de uma pesquisa epidemiológica }\end{array}$ \\
\hline $\mathrm{O}$ & Outcome & Prevalência \\
\hline
\end{tabular}

Fonte: Dados da pesquisa (2021).

A busca de dados ocorreu nas bases: MEDLINE/PUBMED (United States National Library of Medicine), WEB OF SCIENCE E SCOPUS, sendo estas escolhidas por sua relevância acadêmica para a área da saúde e da enfermagem. Para esgotar as possibilidades de busca, o acesso ocorreu a partir do portal de periódicos da Coordenação de Aperfeiçoamento de Pessoal de Nível Superior (CAPES), por meio do Internet Protocol (IP) da Universidade Federal de Alagoas.

A busca foi realizada no mês de maio de 2021, utilizando os seguintes Descritores em Ciências da Saúde DECS/MESH: (idoso OR Aged), (psicotrópicos OR Psychotropic Drugs, (Prevalência OR Prevalence), considerando a aproximação dos mesmos com o tema em estudo. Durante a busca foi utilizado o operador booleano "AND e $O R$ ".

Foram incluídos estudos primários que abordassem a prevalência da utilização de medicamentos psicotrópicos e os fatores associados ao uso destes, disponíveis na íntegra publicados em português ou inglês nos últimos 5 anos. Foram excluídos artigos que não respondem a questões de pesquisa, teóricos ou revisões, relatos de experiências e de casos, monografias, dissertações, teses, resumos em anais de eventos e capítulos de livro. Os artigos duplicados foram considerados apenas uma vez.

Assim, procedeu-se à leitura dos títulos e resumos dos artigos na íntegra para a seleção das publicações de acordo com os critérios de inclusão e exclusão. Em seguida, realizou-se a análise completa dos estudos selecionados, auxiliada por um instrumento que sumarizou as informações dos estudos em título, autores, ano, metodologia, objetivo e principais achados ou conclusão. Este procedimento facilitou o processo de organização e interpretação dos dados.

\section{Resultados e Discussão}

Após os termos combinados foram encontrados no PubMed, Web of Science e Scopus 816 artigos para estudo, a partir da utilização da estratégia de busca, foram encontrados 415 materiais científicos, destes, 124 foram excluídos por repetição, 76 não respondiam à pergunta norteadora da pesquisa e 185 tratavam da utilização por psicofármacos por outros grupos e outras faixas etárias, restando 30 artigos para análise e síntese do conteúdo. A figura 1 a seguir descreve a estratégia 
de busca.

Figura 1: Estratégia de busca. Maceió, 2021.
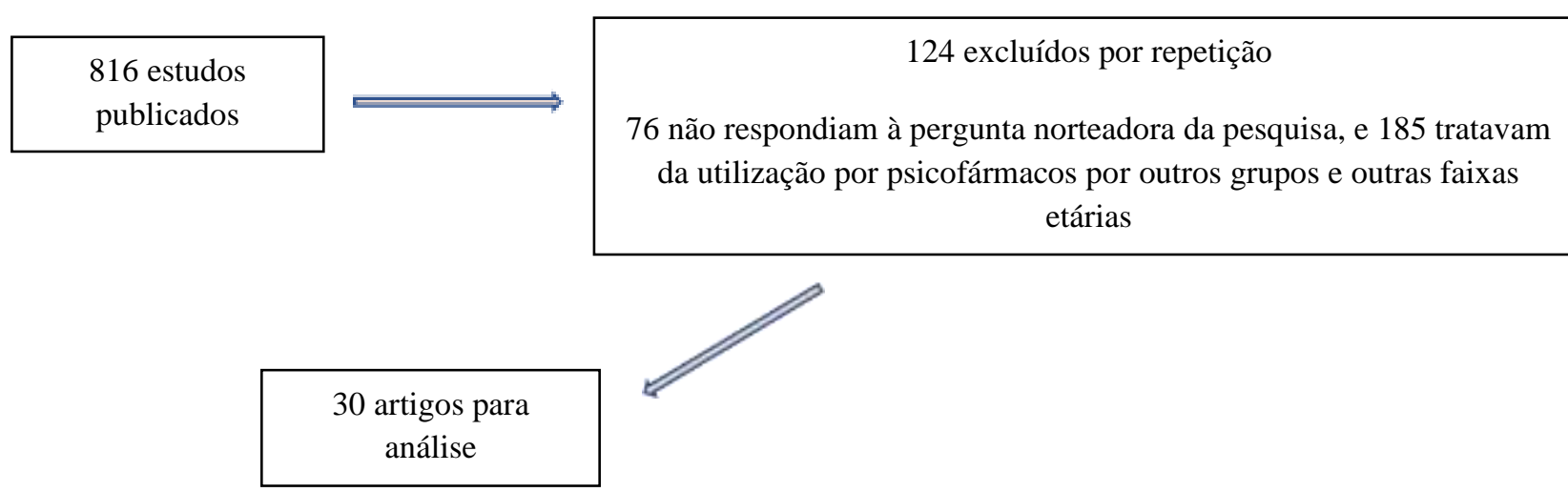

Fonte: Dados da pesquisa (2021).

Organizaram-se, após a seleção, os artigos de acordo com o título, percurso metodológico, periódico e ano e em seguida os principais achados.

A matriz de síntese desta revisão integrativa, exibida na Tabela 1. Descreve aspectos predominantes na produção do conhecimento sobre a relação da utilização e prevalência de medicamentos psicofármacos em pessoas idosas.

Tabela 1: Matriz de síntese dos artigos sobre a prevalência do uso de psicofármacos em idosos de 2016 a 2021.

\begin{tabular}{|c|c|c|c|}
\hline Artigo & Título & Percurso Metodológico & Periódico e Ano \\
\hline 1 & $\begin{array}{l}\text { Depressão, antidepressivos e risco de eventos } \\
\text { cardiovasculares e morte em homens mais } \\
\text { velhos. }\end{array}$ & Estudo de coorte & $\begin{array}{l}\text { Maturitas } \\
2019\end{array}$ \\
\hline 2 & $\begin{array}{l}\text { Prescrição potencialmente inadequada antes e } \\
\text { após o início de medicamentos para demência: } \\
\text { um estudo australiano de base populacional. }\end{array}$ & Estudo de coorte & Geriatr Gerontol Int. 2019. \\
\hline 3 & $\begin{array}{l}\text { Prevalência e padrões de uso de antipsicóticos } \\
\text { e suas associações com saúde mental e } \\
\text { comportamentos problemáticos em idosos com } \\
\text { deficiência intelectual. }\end{array}$ & Estudo transversal & $\begin{array}{l}\text { J Appl Res Intelecto Desabilitado. } \\
2019 .\end{array}$ \\
\hline 4 & $\begin{array}{l}\text { Prevalência e uso persistente de drogas } \\
\text { psicotrópicas em idosos que recebem } \\
\text { atendimento domiciliar no início do estudo. }\end{array}$ & Estudo transversal & BMC Geriatr.2019. \\
\hline 5 & $\begin{array}{l}\text { Uso de antipsicóticos e fatores relacionados } \\
\text { entre pessoas com demência com } 75 \text { anos ou } \\
\text { mais no Japão: Uma estimativa abrangente } \\
\text { baseada na população usando dados médicos e } \\
\text { de cuidados a longo prazo. }\end{array}$ & Estudo transversal & Int J Geriatr Pyschiatry2019. \\
\hline 6 & $\begin{array}{l}\text { Fatores relacionados ao uso de drogas } \\
\text { psicotrópicas potencialmente inapropriadas em } \\
2.343 \text { residentes de } 19 \text { asilos. }\end{array}$ & Estudo transversal & $\begin{array}{l}\text { Geriatr Psychol Neuropsychiatr } \\
\text { Vieil. 1,2018. }\end{array}$ \\
\hline 7 & $\begin{array}{l}\text { Prevalence of use of antipsychotic drugs in the } \\
\text { elderly in Catalonia. }\end{array}$ & Revisão sistemática & Eur. J Clin Pharmacol. 2018 \\
\hline
\end{tabular}


Research, Society and Development, v. 10, n. 11, e09101119228, 2021

(CC BY 4.0) | ISSN 2525-3409 | DOI: http://dx.doi.org/10.33448/rsd-v10i11.19228

\begin{tabular}{|c|c|c|c|}
\hline 8 & $\begin{array}{l}\text { Prevalência e preditores do uso de } \\
\text { antidepressivo tricíclico em idosos coreanos } \\
\text { em clínicas de cuidados primários e } \\
\text { especializados. }\end{array}$ & Estudo transversal & Int J Clin Pharmacol Ther, 2018. \\
\hline 9 & $\begin{array}{l}\text { Prescrições potencialmente inapropriadas para } \\
\text { idosos que tomam antidepressivos: ferramentas } \\
\text { comparativas. }\end{array}$ & Estudo Transversal & BMC Geriatrics, 2018. \\
\hline 10 & $\begin{array}{l}\text { Medicamentos psicotrópicos em idosos em } \\
\text { instituições de cuidados residenciais e } \\
\text { associações com qualidade de vida: um estudo } \\
\text { transversal. }\end{array}$ & Estudo Transversal & BMC Geriatrics, 2017. \\
\hline 11 & $\begin{array}{l}\text { Polifarmácia antipsicótica em } \quad \text { idosos } \\
\text { australianos. }\end{array}$ & Estudo Transversal & Int Psychogeriatr,2017. \\
\hline 12 & $\begin{array}{l}\text { Transtorno bipolar em lares de idosos: impacto } \\
\text { no uso de antipsicóticos, padrões de } \\
\text { diagnóstico e novos diagnósticos em pessoas } \\
\text { com demência. }\end{array}$ & Estudo transversal & Am J Geriatr Psychiatry.2018. \\
\hline 13 & $\begin{array}{l}\text { Engajamento social e uso de antipsicóticos no } \\
\text { tratamento dos sintomas psicológicos e } \\
\text { comportamentais da demência em instituições } \\
\text { de cuidados de longa duração. }\end{array}$ & Estudo Transversal & Can J Nurs Res, 2017. \\
\hline 14 & $\begin{array}{l}\text { Prevalência e características de residentes e } \\
\text { instalações associadas ao uso de antipsicóticos } \\
\text { em unidades de vida assistida, unidades de } \\
\text { cuidados de longa duração: uma análise } \\
\text { transversal de Alberta, Canadá. }\end{array}$ & Estudo Transversal & Envelhecimento de drogas, 2017 \\
\hline 15 & $\begin{array}{l}\text { Uso a longo prazo de antipsicóticos em } \\
\text { pacientes com demência na comunidade: } \\
\text { prevalência e perfil responsável por viés de } \\
\text { tempo não observável devido à hospitalização. }\end{array}$ & Pesquisa por amostragem & Int Clin Psychopharmacol,2017. \\
\hline 16 & $\begin{array}{l}\text { Prevalência, padrões e preditores de } \\
\text { polifarmácia psicotrópica entre idosos com } \\
\text { doença de Parkinson em ambientes de } \\
\text { cuidados de longo prazo nos Estados Unidos. }\end{array}$ & Estudo Transversal & J Parkinsons Dis, 2016. \\
\hline 17 & $\begin{array}{l}\text { Um estudo de prevalência pontual do uso de } \\
\text { medicamentos psicotrópicos em um hospital } \\
\text { geral de agudos. }\end{array}$ & Estudo de Prevalência & Int Psychogeriatr, 2016. \\
\hline 18 & $\begin{array}{l}\text { Prevalência da depressão, ansiedade e stress } \\
\text { numa unidade de saúde familiar do norte de } \\
\text { Portugal. }\end{array}$ & $\begin{array}{c}\text { Estudo transversal } \\
\text { descritivo-correlacional }\end{array}$ & Revista de Enfermagem, 2020. \\
\hline 19 & $\begin{array}{l}\text { Prescrição de medicamentos psicotrópicos em } \\
\text { pacientes com demência: residentes de lares de } \\
\text { idosos versus pacientes que vivem em casa. }\end{array}$ & Estudo Transversal & J Alzheimers Dis,2016. \\
\hline 20 & $\begin{array}{l}\text { Padrões de prescrição de psicotrópicos e } \\
\text { polifarmácia em pacientes idosos } \\
\text { hospitalizados na Irlanda: a influência da } \\
\text { demência na prescrição. }\end{array}$ & Estudo de Observacional & Int Psychogeriatr,2016. \\
\hline 21 & $\begin{array}{l}\text { Prevalência e correlatos do uso de drogas } \\
\text { psicotrópicas em pacientes holandeses em } \\
\text { lares de idosos com demência de início } \\
\text { precoce. }\end{array}$ & Estudo Transversal & Int J Geriatr Psychiatry,2019. \\
\hline
\end{tabular}


Research, Society and Development, v. 10, n. 11, e09101119228, 2021

(CC BY 4.0) | ISSN 2525-3409 | DOI: http://dx.doi.org/10.33448/rsd-v10i11.19228

\begin{tabular}{|c|c|c|c|}
\hline 22 & $\begin{array}{l}\text { Prevalência e uso persistente de drogas } \\
\text { psicotrópicas } \\
\text { atendimento domiciliar no início do estudo. }\end{array}$ & Estudo Longitudinal & BMC Geriatrics, 2018. \\
\hline 23 & $\begin{array}{l}\text { Uso nacional de psicofármacos para } \\
\text { tratamento de depressão autorrelatada na } \\
\text { população adulta urbana brasileira. }\end{array}$ & Estudo transversal & $\begin{array}{l}\text { Revista Brasileira de } \\
\text { Epidemiologia, } 2020 .\end{array}$ \\
\hline 24 & $\begin{array}{l}\text { Medicalization and Indigenous Health: An } \\
\text { analysis of the consumption of psychotropics } \\
\text { by the Xukuru de Cimbres indigenous people. }\end{array}$ & $\begin{array}{l}\text { Estudo descritivo, } \\
\text { quantitativo }\end{array}$ & Ciência \& Saúde Coletiva, 2019. \\
\hline 25 & $\begin{array}{l}\text { Health conditions of institutionalized elderly: } \\
\text { contributions to interdisciplinary action and } \\
\text { health promoter. }\end{array}$ & $\begin{array}{l}\text { Estudo transversal, } \\
\text { descritivo e quantitativo }\end{array}$ & $\begin{array}{l}\text { Cadernos Brasileiros de Terapia } \\
\text { Ocupacional, } 2019 .\end{array}$ \\
\hline 26 & $\begin{array}{l}\text { Tratamento psicotrópico e antidemência em } \\
\text { idosos com sinais clínicos de demência com } \\
\text { corpos de Lewy: um estudo transversal em } 40 \\
\text { lares de idosos na Suécia. }\end{array}$ & $\begin{array}{l}\text { Estudo } \\
\text { farmacoepidemiológico } \\
\text { observacional transversal }\end{array}$ & BMC Geriatrics, 2018. \\
\hline 27 & $\begin{array}{l}\text { Uso persistente de drogas psicotrópicas em } \\
\text { residentes de lares de idosos na Noruega. }\end{array}$ & Estudo longitudinal & BMC Geriatr. 2017. \\
\hline 28 & $\begin{array}{l}\text { Uso de drogas psicotrópicas e mortalidade em } \\
\text { idosos com demência: investigando diferenças } \\
\text { de sexo. }\end{array}$ & $\begin{array}{l}\text { Estudo de coorte } \\
\text { epidemiológico }\end{array}$ & BMC Pharmacol Toxicol. 2017. \\
\hline 29 & $\begin{array}{l}\text { Uso de medicamentos antidepressivos entre } \\
\text { adultos mais velhos em instituições europeias } \\
\text { de cuidados de longo prazo: uma análise } \\
\text { transversal do estudo SHELTER. }\end{array}$ & $\begin{array}{l}\text { Análise transversal dos } \\
\text { dados do estudo SHELTER }\end{array}$ & BMC Geriatr. 2020. \\
\hline 30 & $\begin{array}{l}\text { Início de antipsicóticos após mudança para } \\
\text { instituições residenciais de cuidados para } \\
\text { idosos e mortalidade: um estudo de coorte } \\
\text { nacional. }\end{array}$ & $\begin{array}{l}\text { Estudo de coorte } \\
\text { retrospectivo }\end{array}$ & $\begin{array}{l}\text { Envelhecimento Clin Exp } \\
\text { Res. } 2021 .\end{array}$ \\
\hline
\end{tabular}

Fonte: Dados da pesquisa (2021).

Tabela 2: Principais resultados dos artigos selecionados.

\begin{tabular}{|c|c|}
\hline Artigo & Principais achados \\
\hline 1 & $\begin{array}{l}374 \text { homens }(6,8 \%) \text { tinham um diagnóstico atual ou registrado de depressão e } 365(6,6 \%) \text { estavam em uso de } \\
\text { antidepressivo. }\end{array}$ \\
\hline 2 & $\begin{array}{l}\text { A coorte incluiu } 1.176 \text { pacientes: } 60 \% \text { eram mulheres e a idade média foi de } 80 \text { anos. A prevalência geral de PIP foi de } 85 \% \\
\text { nos } 12 \text { meses antes do início dos medicamentos para demência, em comparação com } 89 \% \text { nos } 12 \text { meses após o início (P } \\
<0,0001 \text { ). }\end{array}$ \\
\hline 3 & $\begin{array}{l}\text { No total, } 45,1 \%(\mathrm{n}=305) \text { possuíam antipsicóticos, e daqueles com uso de antipsicóticos e informações de diagnóstico }(\mathrm{n}= \\
282), 25,9 \%(73) \text { apresentavam transtorno psicótico. } 58 \% \text { das pessoas expostas a antipsicóticos relataram comportamentos } \\
\text { problemáticos. }\end{array}$ \\
\hline 4 & $\begin{array}{l}\text { A prevalência e o uso persistente de drogas psicotrópicas em idosos em atendimento domiciliar foram elevados. Os } \\
\text { participantes com demência usaram mais antipsicóticos e antidepressivos do que os participantes sem demência. }\end{array}$ \\
\hline 5 & $\begin{array}{l}\text { Dos } 25.919 \text { participantes, } 4.865 \text { tinham demência provável e } 1.506 \text { foram prescritos medicamentos antidemencia. A } \\
\text { prevalência de antipsicóticos entre os participantes com provável demência foi de } 10,7 \% \text {, valor menor do que naqueles que } \\
\text { receberam medicamentos antidementosos }(16,4 \%) \text {. }\end{array}$ \\
\hline
\end{tabular}



drogas psicotrópicas $(1,8 \pm 0,9$ drogas psicotrópicas por usuário). Os agentes psicotrópicos representaram 17,4\% dos 18.143 medicamentos utilizados por toda a população do estudo.

Os nossos dados mostram que a prevalência da utilização de PA na Catalunha é consideravelmente mais elevada do que noutros países e que os doentes são expostos durante longos períodos de tempo. Estes resultados podem informar a ação das autoridades reguladoras dos medicamentos e dos gestores dos sistemas de saúde.

TCAs e inibidores seletivos da recaptação de serotonina (ISRSs) representaram 45,2\% e 15,0\% de todas as prescrições de antidepressivos, respectivamente. Os ACTs incluíram $61,5 \%$ e $20,7 \%$ das prescrições de antidepressivos para dor e depressão, respectivamente. medicamentos têm muitos efeitos adversos associados e o uso desses medicamentos deve ser reexaminado ao investigar abordagens para melhorar a qualidade de vida de idosos em cuidados residenciais.

O uso a longo prazo de benzodiazepínicos foi o PIP mais comum reconhecido e o que mais contribuiu significativamente para níveis mais altos de PIP do que outros medicamentos. Os usuários duplos de antipsicóticos tinham maior probabilidade de estar sob os cuidados de um psiquiatra ou de terem sido internados em saúde mental do que aqueles que usavam um único antipsicótico.

Os modelos de regressão identificaram uma série de variáveis relacionadas ao uso de antipsicóticos na ausência de comportamento ou sintomas psicóticos, como transtorno bipolar ( $\mathrm{OR}=5,63$ no CCC; $\mathrm{OR}=5,52$ no LTC), queixas ansiosas $(\mathrm{OR}=1,54$ no LTC para 2,01 em CCC) e errante (OR = 1,83 em ALC).

12 Transtorno bipolar em lares de idosos: impacto no uso de antipsicóticos, padrões de diagnóstico e novos diagnósticos em pessoas com demência.

Cinquenta e seis por cento dos residentes receberam prescrição de algum psicotrópico, 25\% antipsicóticos, 29\% antidepressivos, $15 \%$ ansiolíticos e $13 \%$ hipnóticos, com grandes diferenças entre as unidades. As análises de regressão multinível multivariada revelaram que a prescrição de antipsicóticos era menos provável com maior disponibilidade de médicos e que a prescrição de antidepressivos era mais provável com maior satisfação das enfermeiras em contato com residentes.

A prevalência de uso de psicofármacos na amostra foi de 13,4\%; especificamente, $8,3 \%$ dos indivíduos pesquisados usavam benzodiazepínicos, enquanto $5,0 \%$ usavam antidepressivos. Os seguintes fatores foram independentemente associados ao uso de psicofármacos: gênero feminino $(\mathrm{OR}=2,20$; IC95\% 1,49 - 3,27), diagnóstico médico de depressão $(\mathrm{OR}=6,42$; IC95\% 4,31 - 9,55), 5 ou mais consultas médicas nos últimos 12 meses (OR = 2,15; IC95\% 1,32 - 3,53) e assinatura de plano privado de saúde $(\mathrm{OR}=2,69 ;$ IC95\% 1,86 - 3,88).

Mais de um quarto dos residentes em AL $(26,4 \%)$ e LTC $(31,8 \%)$ estavam em uso de antipsicóticos $(p=0,006)$. A prevalência de uso potencialmente inadequado foi semelhante em AL e LTC (23,4 vs. 26,8\%, p = 0,09). No entanto, entre os usuários, a proporção de uso de antipsicóticos considerada potencialmente inadequada foi significativamente maior em AL do que LTC (AL: 231/287 = 80,5\%; LTC: 224/318 = 70,4\%; $\mathrm{p}=0,004$ ).

16 Este estudo mostrou que o uso prolongado de AP permaneceu frequente em pacientes comunitários com demência. Ele também mostrou que a prevalência de usuários de longo prazo quase dobrou quando os períodos de hospitalização foram contados como exposição a AP. Isso reforça a necessidade de considerar os períodos de internação na avaliação da exposição a medicamentos em populações com períodos frequentes de internação. Os usuários de longo prazo foram mais expostos aos benzodiazepínicos [OR = 1,2, IC 95\% $(1,1-1,4)$.

Entre os idosos residentes em lares de idosos com DP, a prevalência nacionalmente representativa de polifarmácia psicotrópica foi de $26,28 \%$, ao passo que foi de $21,36 \%$ no ambiente domiciliar de saúde. O uso de medicamentos antidepressivos constituiu a maioria do uso de medicamentos psicotrópicos entre os residentes de asilos $(48,91 \%)$ e de saúde domiciliar $(40,98 \%)$ com DP. 
Os prontuários de 197 pacientes foram auditados, 139 (70\%) tinham 65 anos ou mais. Oitenta e nove (45\%) dos pacientes receberam prescrição de psicotrópico, sendo 35 (17,7\%) antidepressivos; 21 (11\%) antipsicóticos; 19 (10\%) benzodiazepinas; seis (3\%) estabilizador de humor; cinco (2\%) inibidores da colinesterase; um (0,5\%) medicamento antiparkinsoniano e um (1\%) paciente em uso de zolpidem e outro paciente com melatonina (1\%). A maioria das prescrições $(72 \%)$ foi para pessoas com 65 anos ou mais e 27 (20\%) das indicações foram consideradas prescrições off label. Houve déficit na documentação das indicações de uso de psicofármacos e no acompanhamento.

Com uma amostra de 207 usuários por meio de amostragem não probabilística e de conveniência. Depressão, ansiedade e estresse foram mais prevalentes em indivíduos com idade superior a 65 anos, aqueles que percebiam sua saúde como ruim e aqueles que usavam psicotrópicos.

20 Dos 50.932 pacientes com demência cadastrados no BNA, 40,1\% tinham pelo menos um psicotrópico prescrito. A maioria dos pacientes tratados com pelo menos uma classe de psicofármacos fazia uso de antidepressivos $(69,0 \%)$, qualquer que fosse o tipo de residência, e 16,1\% eram tratados com antipsicóticos.

21 Dos 149 pacientes com diagnóstico de demência, apenas 53 tinham diagnóstico prévio. Na admissão hospitalar, 458/583 pacientes experimentaram polifarmácia ( $\geq 5$ medicamentos). Pessoas com demência $(\mathrm{PcD})$ eram significativamente mais propensas a serem prescritas pelo menos um medicamento psicotrópico do que pacientes sem demência (99/147 vs. $182 / 436 ; \mathrm{p}<0,001)$.

22 Oitenta e sete por cento dos residentes usavam psicofármacos e $47 \%$ usavam dois ou mais psicofármacos. Não foram encontradas associações significativas com idade, sexo, subtipo de demência e gravidade da demência.

23 No total, 45,1\% ( $\mathrm{n}=305)$ faziam uso de antipsicóticos, e daqueles com uso de antipsicóticos e informações diagnósticas (n $=282$ ), 25,9\% (73) apresentavam transtorno psicótico. 58\% das pessoas expostas aos antipsicóticos relataram comportamentos problemáticos.

24 Os achados revelam uma população adulta vulnerável do ponto de vista socioeconômico, um padrão de cronificação do consumo dos psicotrópicos e o distanciamento das práticas de curas tradicionais indígenas, característicos do processo de medicalização da saúde. fazem uso de psicofármacos.

26 Uma pesquisa com povos Xukuru de Pesqueira, PE, revela que 26,67\% das pessoas que utilizam psicotrópicos são idosos, e faz uso dos benzodiazepínicos e outros psicotrópicos. O estudo ainda revela uma assistência a saúde mental fragmentada focada na doença e no uso da medicação.

27 Idosos institucionalizados entre 60 e 105 anos, fazem uso, excessivo de medicamentos psicotrópicos. A maioria na faixa etária dos 70 anos.

Cerca de $43,8 \%$ dos pacientes receberam medicamentos regular potencialmente inadequado e $9,9 \%$ receberam regularmente três ou mais medicamentos potencialmente inadequados. $92,7 \%$ dos residentes receberam medicamentos que necessitavam de vigilância e $69,7 \%$ dos idosos receberam pelo menos um medicamento psicotrópico regularmente. O uso de drogas psicoativas aumentou o número de quedas no episódio agudo de uma infecção ou quadro de desidratação.

29 Drogas psicotrópicas são frequentemente usadas como tratamento de longo prazo entre os residentes do NH e estão associadas à gravidade dos sintomas neuropsiquiátricos, mas não à gravidade da demência.

30 As drogas psicotrópicas são comuns entre os idosos com demência e têm sido associadas ao aumento da mortalidade. Foram observadas diferenças entre os sexos no risco de mortalidade associado ao uso de antidepressivos e benzodiazepínicos, destacando a necessidade de uma investigação mais aprofundada sobre o impacto do sexo.

Fonte: Dados da pesquisa (2021).

Idosos podem ter doenças mentais ou comportamentais, algumas das quais podem até estar relacionadas a seus próprios fatores moduladores da idade, como demência, depressão e ansiedade (Santos, et al., 2020). No estudo de 
Lornstad, Aarøen, Bergh, Benth, \& Helvik (2019) é relatado que idosos domiciliados acabam recebendo uma maior prevalência de medicamentos psicotrópicos, com destaque para aqueles com demência. Onde o tipo mais comum de psicotrópicos utilizado nesta amostra foram os sedativos, com prevalência estimada de 21 a $23 \%$. No Canadá em vários departamentos de saúde e jurisdições canadenses, os idosos com demência geralmente usam medicamentos antipsicóticos. Sendo ainda mais frustrante a prevalência do uso entre indivíduos que não apresentam sintomas comportamentais e / ou psicóticos (Rios, Perlman, Costa., Heckman, Hirdes, \& Mitchell (2017).

Foram ainda observadas diferenças entre os sexos no risco de mortalidade associado ao uso de antidepressivos e benzodiazepínicos, destacando a necessidade de uma investigação mais aprofundada sobre o impacto do sexo. Estudos ainda revelam que drogas psicotrópicas entre os idosos com demência, têm sido associadas ao aumento da mortalidade.

As drogas psicotrópicas são importantes para o tratamento do sofrimento humano. No entanto, seu uso não deve ser rigoroso, mas deve ser combinado com cuidados mais extensos, e uma interface maior entre a medicação e a psicoterapia que deve ser estabelecida para fornecer uma ajuda mais eficaz (Prado et al., 2017). Porém Saleh, Penning, Cloutier, Mallidou, Nuernberger, \& Taylor (2017) em seu estudo relata que quando usados com cautela, os antipsicóticos podem melhorar a saúde física e mental dos idosos, aumentando assim seus níveis de engajamento social. Por outro lado, os antipsicóticos têm efeito sedativo e aumentam os riscos à saúde podendo ter um impacto negativo em suas habilidades sociais.

Porém os resultados dos estudos confirmaram que a história de diagnóstico médico de depressão é o fator mais relacionado ao uso de psicofármacos, além do uso de tratamentos para demência e Alzheimer. Um achado interessante segundo Saleh et. al., (2017) é de que residentes de instituições de cuidados de longa permanência em cerca de 31, $8 \%$ não apresentavam nenhum perfil de agressividade e mesmo assim receberam medicamento antipsicóticos, enquanto 27,5\% desses residentes com quadro de agressividade severa de comportamento não recebeu antipsicóticos. Outras características de outros estudos estão: mulheres com maior número de atendimentos médicos. Benzodiazepínicos (em termos de farmacologia) e bromazina (em termos de ingrediente ativo) são os principais psicotrópicos.

Diante dessa situação, o uso de psicofármacos nessa população tornou-se uma prática rotineira, sendo esses medicamentos os mais prescritos entre os idosos. Devido à maior incidência de episódios depressivos nessas pessoas, os antidepressivos estão entre as principais prescrições. Nas prescrições para o sono, os benzodiazepínicos também dominam, pois os distúrbios do sono são as principais causas da maioria das queixas nessa população (Pancote, Sasaki, Santos \& Soler, 2018).

Contudo a utilização concomitante de múltiplos medicamentos pode acabar prejudicando a saúde com desfechos indesejáveis e aumento de reações adversas, diminuição funcional da capacidade e declínio cognitivo do idoso, e menor adesão à terapia medicamentosa (Almeida, Reinrs, Azevedo, Silva, Cardoso \& Souza, 2017). No entanto deve-se estabelecer que os medicamentos considerados não tão seguros para os idosos, necessitam que suas prescrições precisam ser evitadas (Bueno, Almeida \& Rocha, 2016).

\section{Considerações Finais}

As principais dificuldades e estratégias encontras no presente estudo foi semelhante a vários estudos que relatam a relação entre a utilização dos medicamentos psicotrópicos, onde a grande demanda se encontra associada com a depressão e a baixa qualidade de vida.

Além das dificuldades que os idosos já enfrentam com fatores da própria idade, acabam corroborando com a compreensão sobre os tipos de medicamentos psicofármacos, tempo de duração do tratamento, e a fatores associados que acabam levando a consumação. Como abandono da família, vivência em lares de idosos, depressão e outros transtornos mentais, tem servido como base para a utilização desses fármacos, além de novas indicações terapêuticas de psicofármacos já existentes. 
Observa-se que a melhor conduta para o tratamento dos transtornos mentais e a utilização dos psicofármacos, deve ser sempre ponderada aos riscos-benefícios que possa justificar o seu consumo e os seus recursos altamente explorados, sempre que necessário acompanhada de um profissional treinado e capacitado.

Sugere-se para pesquisas futuras, estudos de base populacional para avaliar a frequência do uso de substâncias psicotrópicas na população idosa, bem como a avaliação dos efeitos desses medicamentos associados com outros fármacos. Além de padrões sobre usos de medicamentos antipsicóticos.

\section{Referências}

Ackel M. M. A., Costa M. F. L., Costa C. E. \& Filho A. I. L. (2017). Uso de psicofármacos entre idosos residentes em comunidade: prevalência e fatores associados. Rev. bras. epidemiol. São Paulo, v. 20, n. 1, p. 57-69.

Almeida N. A., Reinrs A. A. O, Azevedo R. C. S., Silva A. M. C., Cardoso J. D. C. \& Souza L.C. (2017). Prevalência e fatores associados à polifarmácia entre os idosos residentes na comunidade. Rev. bras. geriatr. gerontol., p. 138-148.

Asensio, C., Escoda, N., Sabaté, M., Carbonell, P., López, P., \& Laporte, J.-R. (2018). Prevalence of use of antipsychotic drugs in the elderly in Catalonia. European Journal of Clinical Pharmacology, 74(9), 1185-1186. https://doi.org/10.1007/s00228-018-2469-6.

Barbosa, V. F. B., Cabral, L. B., \& Alexandre, A. C. S. (2019). Medicalização e Saúde Indígena: uma análise do consumo de psicotrópicos pelos índios Xukuru de Cimbres. Ciência \& Saúde Coletiva, 24(8), 2993-3000. https://doi.org/10.1590/1413-81232018248.22192017.

Boucherie, Q., Gentile, G., Chalançon, C., Sciortino, V., Blin, O., Micallef, J., \& Bonin-Guillaume, S. (2017). Long-term use of antipsychotics in communitydwelling dementia patients. International Clinical Psychopharmacology, 32(1), 13-19. https://doi.org/10.1097/yic.0000000000000150.

Bhattacharjee, S., Goldstone, L., \& Warholak, T. (2016). Prevalence, Patterns and Predictors of Psychotropic Polypharmacy Among Elderly Individuals with Parkinson's Disease In Long Term Care Settings In The United States. Journal of Parkinson's Disease, 6(1), 247-255. https://doi.org/10.3233/jpd-150646.

Brännström, J., Boström, G., Rosendahl, E., Nordström, P., Littbrand, H., Lövheim, H., \& Gustafson, Y. (2017). Psychotropic drug use and mortality in old people with dementia: investigating sex differences. BMC Pharmacology and Toxicology, 18(1). https://doi.org/10.1186/s40360-017-0142-9

Brunero, S., Wand, A. P. F., Lamont, S., \& John, L. (2016). A point prevalence study of the use of psychotropic medication in an acute general hospital. International Psychogeriatrics, 28(6), 967-975. https://doi.org/10.1017/s104161021500232x

Bueno D., Almeida T. T., \& Rocha B., S. (2016). Prevalência de prescrição de medicamentos potencialmente inapropriados para idosos em uma unidade de saúde da família de Porto Alegre/RS. Rev. APS. 19(3): 370 - 375.

Carnahan, R. M., \& Letuchy, E. M. (2018). Bipolar Disorder in Nursing Homes: Impact on Antipsychotic Use, Diagnosis Patterns, and New Diagnoses in People with Dementia. The American Journal of Geriatric Psychiatry, 26(1), 2-10. https://doi.org/10.1016/j.jagp.2017.09.007.

Chyczij, F. F., Ramos, C., Santos, A., Jesus, L., \& Alexandre, J. (2020). Prevalência da depressão, ansiedade e stress numa unidade de saúde familiar do norte de Portugal. Revista de Enfermagem Referência, V Série (No 2). https://doi.org/10.12707/riv19094.

Crossetti M. G. O. Revisão integrativa de pesquisa na enfermagem o rigor científico que lhe é exigido. Rev. Gaúcha Enferm.; 33(2):8-9.

Diniz A., Pillon S.C., Monteiro S., Pereira A., Gonçalves J., \& Santos M. A. (2017). Uso de substâncias psicoativas em idosos, São Paulo, SP, maio-ago. Revista Psicologia: Teoria e Prática 19(2), 23-41.

European Monitoring Centre for Drug Addiction. (2010). Treatment and care for older drug users. Luxemburgo: Publications Office of the European Union. https://www.emcdda.europa.eu/publications/selected-issues/older-drug-users_en

Eshetie, T. C., Nguyen, T. A., Gillam, M. H., \& Kalisch Ellett, L. M. (2019). Potentially inappropriate prescribing before and after initiation of medicines for dementia: An Australian population-based study. Geriatrics \& Gerontology International. https://doi.org/10.1111/ggi.13686.

Fulone, I., \& Lopes, L. C. (2017). Potentially inappropriate prescriptions for elderly people taking antidepressant: comparative tools. BMC Geriatrics, 17(1). https://doi.org/10.1186/s12877-017-0674-2

Greenhalgh T. Como ler artigos científicos: fundamentos da medicina baseada em evidências. 2a Ed. Porto Alegre: Artmed; 2005.

Harrison, S. L., Bradley, C., Milte, R., Liu, E., Kouladjian O’Donnell, L., Hilmer, S. N., \& Crotty, M. (2018). Psychotropic medications in older people in residential care facilities and associations with quality of life: a cross-sectional study. BMC Geriatrics, 18(1). https://doi.org/10.1186/s12877-018-0752-0.

Helvik, A.-S., Šaltytė Benth, J., Wu, B., Engedal, K., \& Selbæk, G. (2017). Persistent use of psychotropic drugs in nursing home residents in Norway. BMC Geriatrics, 17(1). https://doi.org/10.1186/s12877-017-0440-5.

Hwang, J. E., Song, I., Lee, E.-K., Ha, D., \& Shin, J.-Y. (2018). Prevalence and predictors of tricyclic antidepressant use among elderly Koreans in primarycare and specialty clinics. Int. Journal of Clinical Pharmacology and Therapeutics, 56(05), 224-230. https://doi.org/10.5414/cp203157.

Jacquin-Piques, A., Sacco, G., Tavassoli, N., Rouaud, O., Bejot, Y., Giroud, M., Robert, P., Vellas, B., \& Bonin-Guillaume, S. (2016). Psychotropic Drug Prescription in Patients with Dementia: Nursing Home Residents Versus Patients Living at Home. Journal of Alzheimer's Disease, 49(3), 671-

680. https://doi.org/10.3233/jad-150280. 
Kalisch Ellett, L. M., Pratt, N. L., Kerr, M., \& Roughead, E. E. (2017). Antipsychotic polypharmacy in older Australians. International Psychogeriatrics, 30(4), 539-546. https://doi.org/10.1017/s1041610217001934

Kuroda, N., Hamada, S., Sakata, N., Jeon, B., Iijima, K., Yoshie, S., Ishizaki, T., Jin, X., Watanabe, T., \& Tamiya, N. (2018). Antipsychotic use and related factors among people with dementia aged 75 years or older in $\mathrm{J}$ apan: A comprehensive population-based estimation using medical and long-term care data. International Journal of Geriatric Psychiatry, 34(3), 472-479. https://doi.org/10.1002/gps.5041.

Lornstad, M. T., Aarøen, M., Bergh, S., Benth, J. Š., \& Helvik, A.-S. (2019). Prevalence and persistent use of psychotropic drugs in older adults receiving domiciliary care at baseline. BMC Geriatrics, 19(1). https://doi.org/10.1186/s12877-019-1126-y

Mendes K. D. S., Silveira R. C. C. P., \& Galvão C. M. (2019). Uso de gerenciador de referências bibliográficas na seleção dos estudos primários em revisão integrativa. Texto contexto - enferm., v. 28: e20170204.

Mittelmeier, J., Edwards, R. L., Davis, S. K., Nguyen, Q., Murphy, V. L., Brummer, L., \& Rienties, B. (2018). https://doi.org/10.14786/flr.v6i2. Frontline Learning Research, 20-38. https://doi.org/10.14786/flr.v6i2.348.

Mulders, A. J. M. J., Zuidema, S. U., Leeuwis, R., Bor, H., Verhey, F. R. J., \& Koopmans, R. T. C. M. (2019). Prevalence and correlates of psychotropic drug use in Dutch nursing home patients with young-onset dementia. International Journal of Geriatric Psychiatry, 34(8), 1185-

1193. https://doi.org/10.1002/gps.5116

Nascimento L.C.S, Salvi J. O. (2018). Ansiedade, depressão e medicamentos psicotrópicos em idosos institucionalizados no municipio de ji-paraná, rondônia. Brazilian Journal of Surgery and Clinical Research V.21, n.3, pp.38-42.

Nasser, F. J., Almeida, M. M. d., Silva, L. S. d., Almeida, R. G. P. d., Barbirato, G. B., Mendlowicz, M. V., \& Mesquita, C. T. (2016). Psychiatric Disorders and Cardiovascular System: Heart-Brain Interaction. International Journal of Cardiovascular Sciences, 29(1). https://doi.org/10.5935/2359-4802.20160003

Noia A., Sivia R. S., Yeda A. O. D., Maria L.L., \& Nicolina S. R. L. (2012). Fatores associados ao uso de psicotrópicos por idosos residentes no Município de São Paulo. Rev. esc. enferm. USP, São Paulo, v. 46, n. spe, p. 38-43.

O'Dwyer, C., McCallion, P., Henman, M., McCarron, M., O'Leary, E., Burke, E., O'Connell, J., \& O'Dwyer, M. (2019). Prevalence and patterns of antipsychotic use and their associations with mental health and problem behaviours among older adults with intellectual disabilities. Journal of Applied Research in Intellectual Disabilities, 32(4), 981-993. https://doi.org/10.1111/jar.12591.

Prado M. A. M. B., Francisco P. M. S. B. \& Barros M. B. A. (2017) Uso de medicamentos psicotrópicos em adultos e idosos residentes em Campinas, São Paulo: um estudo transversal de base populacional. Epidemiol. Serv. Saúde.:26(4): 747-758.

Pancote C. G., Sasaki N. S. G. M. S., Santos M. L. S. G., \& Soler Z. A. S. G. (2018). Envelhecimento e uso de psicotrópicos. Enfermagem Brasil ;17(5):42642.

Prudent, M., Parjoie, R., Jolly, D., Dramé, M., Badr, S., Novella, J.-L., \& Mahmoudi, R. (2018). Factors related to use of potentially inappropriate psychotropic drugs in 2,343 residents of 19 nursing homes. Gériatrie et Psychologie Neuropsychiatrie du Viellissement, 16(3), 279285. https://doi.org/10.1684/pnv.2018.0742.

Ramos, L. R., Mari, J. d. J., Fontanella, A. T., Pizzol, T. d. S. D., Bertoldi, A. D., \& Mengue, S. S. (2020b). Nationwide use of psychotropic drugs for treatment of self-reported depression in the Brazilian urban adult population. Revista Brasileira de Epidemiologia, 23. https://doi.org/10.1590/1980549720200059 .

Rios, S., Perlman, C. M., Costa, A., Heckman, G., Hirdes, J. P., \& Mitchell, L. (2017). Antipsychotics and dementia in Canada: a retrospective cross-sectional study of four health sectors. BMC Geriatrics, 17(1). https://doi.org/10.1186/s12877-017-0636-8.

Saleh, N., Penning, M., Cloutier, D., Mallidou, A., Nuernberger, K., \& Taylor, D. (2017). Social Engagement and Antipsychotic Use in Addressing the Behavioral and Psychological Symptoms of Dementia in Long-Term Care Facilities. Canadian Journal of Nursing Research, 49(4), 144152. https://doi.org/10.1177/0844562117726253

Santos, J. M. S, Viera B. A. G, Santana E. J., Caldas M. A. G, Canuto P. C. O. V., Araújo R. J. S \& Lopes R. F. (2020). Idosos e o uso desordenado de psicofármaco na atenção básica. Braz. J. Hea. Rev., Curitiba, v. 3, n. 2, p.1901-1908.

Santos C. M. C, Pimenta C. A. M, \& Nobre M. R. C. (2007). The PICO strategy for the research question construction and evidence searches. Rev Lat Am Enfermagem; 15(3):508-11.

Sales A. S, Sales M. G. S. \& Casotti C.A. (2017). Perfil farmacoterapêutico e fatores associados à polifarmácia entre idosos de Aiquara, Bahia, em 2014. Epidemiol. Serv. Saúde; 26(1): 121-132.

Secoli S. R, Marquesini E. A, FabrettiI S.C, Corona L.P \& Lieber N.S.R (2018). Tendência da prática de automedicação entre idosos brasileiros entre 2006 e 2010. Revista Brasileira de Epidemiologia [online]. v. 21, n. Suppl 02.

Silva, R. S. d., Fedosse, E., Pascotini, F. d. S., \& Riehs, E. B. (2019). Condições de saúde de idosos institucionalizados: contribuições para ação interdisciplinar e promotora de saúde. Cadernos Brasileiros de Terapia Ocupacional, 27(2), 345-356. https://doi.org/10.4322/2526-8910.ctoao1590

Stock, K. J., Amuah, J. E., Lapane, K. L., Hogan, D. B., \& Maxwell, C. J. (2016). Prevalence of, and Resident and Facility Characteristics Associated With Antipsychotic Use in Assisted Living vs. Long-Term Care Facilities: A Cross-Sectional Analysis from Alberta, Canada. Drugs \& Aging, 34(1), 3953. https://doi.org/10.1007/s40266-016-0411-0.

Zahirovic, I., Torisson, G., Wattmo, C., \& Londos, E. (2018). Psychotropic and anti-dementia treatment in elderly persons with clinical signs of dementia with Lewy bodies: a cross-sectional study in 40 nursing homes in Sweden. BMC Geriatrics, 18(1). https://doi.org/10.1186/s12877-018-0740-4 
Research, Society and Development, v. 10, n. 11, e09101119228, 2021

(CC BY 4.0) | ISSN 2525-3409 | DOI: http://dx.doi.org/10.33448/rsd-v10i11.19228

Walsh, K. A., O'Regan, N. A., Byrne, S., Browne, J., Meagher, D. J., \& Timmons, S. (2016). Patterns of psychotropic prescribing and polypharmacy in older hospitalized patients in Ireland: the influence of dementia on prescribing. International Psychogeriatrics, 28(11), 1807-

1820. https://doi.org/10.1017/s1041610216001307.

Wolf, I.-K., Du, Y., \& Knopf, H. (2017). Changes in prevalence of psychotropic drug use and alcohol consumption among the elderly in Germany: results of two National Health Interview and Examination Surveys 1997-99 and 2008-11. BMC Psychiatry, 17(1). https://doi.org/10.1186/s12888-017-1254-x. 